\title{
Geodesically Complete Lorentzian Metrics on Some Homogeneous 3 Manifolds
}

\author{
Shirley BROMBERG ${ }^{\dagger}$ and Alberto MEDINA $\ddagger$ \\ † Departameto de Matemáticas, UAM-Iztapalapa, México \\ E-mail: stbs@xanum.uam.mx \\ $\ddagger$ Département des Mathématiques, Université de Montpellier II, UMR, CNRS, \\ 5149, Montpellier, France \\ E-mail: medina@math.univ-montp2.fr
}

Received June 24, 2008, in final form December 10, 2008; Published online December 18, 2008

Original article is available at http://www.emis.de/journals/SIGMA/2008/088/

\begin{abstract}
In this work it is shown that a necessary condition for the completeness of the geodesics of left invariant pseudo-Riemannian metrics on Lie groups is also sufficient in the case of 3-dimensional unimodular Lie groups, and not sufficient for 3-dimensional non unimodular Lie groups. As a consequence it is possible to identify, amongst the compact locally homogeneous Lorentzian 3-manifolds with non compact (local) isotropy group, those that are geodesically complete.
\end{abstract}

Key words: Lorentzian metrics; complete geodesics; 3-dimensional Lie groups, Euler equation

2000 Mathematics Subject Classification: 53C22; 53C50; 57M50; 22E30

\section{Introduction}

The aim of this paper is to characterize the geodesically complete left invariant Lorentzian metrics on unimodular 3-dimensional Lie groups. The main result (Theorem 2) improve and complete Proposition 3.2 given in [1]. It is well known that an unimodular Lie group of dimension 3 contains an infinity of cocompact discret subgroups. Consequently our results provide a rich class of compact and complete (or incomplete) Lorentzian 3-manifolds. In particular, we obtain the geodesically complete and compact locally homogeneous Lorentz 3-manifolds with non compact (local) isotropy group as described in [3]. Moreover, our results give a correct description of the complete (or incomplete) Lorentzian metrics on $M=\Gamma \backslash \operatorname{PSL}(2, \mathbb{R})$, where $\Gamma$ is a Fuchsian group, obtained from a left invariant Lorentzian metric on $\operatorname{PSL}(2, \mathbb{R})$.

To characterize the complete left invariant pseudo-Riemannian metrics which are not definite on arbitrary Lie groups is a difficult problem. On one side of the spectrum we find groups where all such metrics are complete, such as the Abelian groups, the compact groups, the 2-nilpotent groups [5] or the group of isometries of the Euclidean plane, and on the other side, groups such as the group of the affine transformations of the line in which no Lorentzian metric is complete.

The structure of this work is as follows: In Section 2 the definitions and some general results are given, particularly the algebraic counterparts of the geometric properties of left invariant pseudo-Riemannian metrics defined on Lie groups. In Section 3 we characterize the left invariant metrics on 3-dimensional unimodular Lie groups that are geodesically complete. The following result is proved:

A left invariant metric on an unimodular 3-dimensional Lie group is geodesically complete if and only if all geodesics that run on 1-parameter subgroups are complete.

In Section 4 we give an example that shows that Theorem 2 is not valid without the hypothesis of unimodularity. We also show that previous results in the literature [4] are not accurate. 


\section{Preliminaries and general results}

Let $\mathcal{G}=\mathrm{T}_{\epsilon}(G)$ be the Lie algebra of a Lie group $G$, where $\epsilon$ stands for the identity of $G$. Every left invariant pseudo-Riemannian metric $q$ on $G$ induces a left invariant connection $\nabla$ on $G$, the Levi-Civita connection; a non degenerate quadratic form $\langle\cdot, \cdot\rangle$ on $\mathcal{G}$, the polar form of $q_{\epsilon}$, the restriction of $q$ to $\mathcal{G}$; and a product $x \cdot y$ on $\mathcal{G}$ defined by

$$
(x \cdot y)^{+}=\nabla_{x^{+}} y^{+}
$$

where $x^{+}$is the left invariant vector field on $G$ determined by $x \in \mathcal{G}$. These objects are related by the Koszul formula:

$$
\langle x \cdot y, z\rangle=\frac{1}{2}(\langle[x, y], z\rangle-\langle[y, z], x\rangle+\langle[z, x], y\rangle) .
$$

If $c(t)$ is a curve in $G$ and

$$
x(t)=\left(L_{c(t)}\right)_{*, c(t)}^{-1} c^{\prime}(t),
$$

where $L_{\sigma}$ denotes the left multiplication by $\sigma$ in $G$, it follows that $c(t)$ is a geodesic if and only if $x(t)$ is a solution of the equation

$$
\dot{x}=-x \cdot x .
$$

That $\nabla$ is geodesically complete is equivalent to the completeness of the vector field given by equation (1). Clearly the energy, that is $q_{\epsilon}(x)=\langle x, x\rangle$, is a first integral of equation (1).

Notice that if $G$ is Abelian, then $x \cdot y \equiv 0$ for every left invariant pseudo-Riemannian metric. Hence, every left invariant pseudo-Riemannian metric on an Abelian Lie group is complete.

If $\phi: \mathcal{G} \rightarrow \mathcal{G}^{*}$ is the canonical linear isomorphism associated to $\langle\cdot, \cdot\rangle$ then the equation (1) can be written, in terms of the co-adjoint representation as

$$
\dot{\xi}=-\operatorname{ad}_{\phi^{-1} \xi}^{*} \xi
$$

with $\xi \in \mathcal{G}^{*}$. Equation (2) is called the Euler equation associated to the pseudo-Riemannian metric, and the corresponding vector field is called the Euler vector field. Consequently the solutions of equation (2) are curves in the orbits of the co-adjoint representation of $G$. This implies that (2) is complete if $G$ is compact. The energy on $\mathcal{G}^{*}$ is given by $q_{\epsilon}\left(\phi^{-1} \xi\right)$.

Suppose that $(G, \mathbf{k})$ is orthogonal or quadratic, i.e. $\mathbf{k}$ is a bi-invariant pseudo-Riemannian metric. Then $x \cdot x=0$ and equation (1) is obviously complete and the geodesics through $\epsilon$ are the 1-parameter subgroups of $G$. Note that every left invariant pseudo-Riemannian metric $\langle\cdot, \cdot\rangle$ on a orthogonal Lie group $(G, \mathbf{k})$ is given by the formula $\langle x, y\rangle=\mathbf{k}(u(x), y)$ where $u$ is a $\mathbf{k}$-symmetric linear isomorphism of $\mathcal{G}$. Equation (1) is given in this case by

$$
u(\dot{x})=[x, u(x)]
$$

which is equivalent by a linear change of coordinates to the Lax pair

$$
\dot{x}=\left[x, u^{-1} x\right] .
$$

Equation (3) has two quadratic first integrals $\mathbf{k}(x, x), \mathbf{k}\left(x, u^{-1} x\right)$.

The vector fields induced by a left invariant pseudo-Riemannian metric are special cases of homogeneous quadratic vector fields.

Definition 1. A homogeneous quadratic vector field on the $n$-dimensional affine space $\mathbb{R}^{n}$ is a vector field $F: \mathbb{R}^{n} \rightarrow \mathbb{R}^{n}, F=\left(F_{1}, \ldots, F_{n}\right)$, where $F_{i}$ is a homogeneous polynomial of degree 2 , $i=1, \ldots, n$. If the solutions of the differential equation $\dot{X}=F(X)$ are defined for all $t \in \mathbb{R}$, the vector field $F$ is called complete. 
The following definition provides the simplest example of complete homogeneous quadratic vector fields:

Definition 2. An affine-quadratic (or linearizable) vector field is a homogeneous quadratic vector field such that $\dot{X}=F(X)$ is equivalent by a linear change of coordinates to

$$
\dot{U}=A(V) U+B(V), \quad \dot{V}=0,
$$

where $A$ is linear and the coordinate functions of $B$ are homogeneous polynomials of degree 2 .

It is easily checked that every affine-quadratic vector field is complete.

Definition 3. Let $F$ be a homogeneous quadratic vector field. We define

$$
\mathcal{I}:=\{X \neq 0 ; X, F(X) \text { are linearly dependent }\} .
$$

If $X \in \mathcal{I}$ and $F(X)=0, X$ is called a zero of $F$. If $X \in \mathcal{I}$ and $F(X) \neq 0$ then $X$ is called a relaxed idempotent of $F$.

In fact a strict idempotent is a non trivial solution of $F(X)=X$. If $X \in \mathcal{I}$ and $F(X) \neq 0$, then there exists $\lambda \neq 0$ such that $F(\lambda X)=\lambda X$, i.e. for every relaxed idempotent there is a strict idempotent in the same direction (the line through $X$ is invariant by $F$ ). In what follows we make no distinction between relaxed or strict idempotents.

Remark 1. A simple calculation shows that any idempotent of $F$ gives an incomplete solution for $\dot{X}=F(X)$.

In fact the solution of $\dot{X}=F(X)$ with initial condition an idempotent $X_{0}$ is given by $t \mapsto \alpha(t) X_{0}$ where $\alpha$ satisfies $\dot{\alpha}=\alpha^{2}$. Moreover if $X_{0} \in \mathcal{I}$ for the Levi-Civita product, the geodesic through $\epsilon$ with velocity $X_{0}$ is given by $t \mapsto \exp \beta(t) X_{0}$, where $\beta$ is such that $\beta(0)=0$, $\dot{\beta}(t)=\alpha(t)$ and $\dot{\alpha}=\alpha^{2}$. A result by Kaplan and Yorke (see [7]) states that for every quadratic homogeneous vector field $F, \mathcal{I} \neq \varnothing$, hence

Proposition 1. Any left invariant pseudo-Riemannian metric on $G$ has a geodesic that runs on a 1-parameter subgroup. Furthermore if the pseudo-Riemannian metric is complete then the corresponding Levi-Civita product has a zero. Hence for such metrics, there is a 1-parameter subgroup of $G$ which is a geodesic.

Lemma 1. Let $X$ be an idempotent of a quadratic vector field $F$ and $Q$ a quadratic form which is a first integral of $\dot{X}=F(X)$. Then $Q(X)=0$.

Proof. Let $P$ be the bilinear polar form associated to $Q$. Then for any curve $t \mapsto X(t)$

$$
\frac{d}{d t} Q(X)=2 P\left(X, \frac{d}{d t} X\right)
$$

If $t \mapsto X(t)$ is a solution of $\dot{X}=F(X)$ and $Q$ is a first integral, then

$$
0=\frac{d}{d t} Q(X)=2 P\left(X, \frac{d}{d t} X\right)=2 P(X, F(X))=-2 Q(X)
$$

as was asserted.

The following theorem is a general result of completeness for homogenous quadratic vector fields in dimension 2 . 
Theorem 1 ([2]). Let $F$ be a homogeneous quadratic vector field on the plane. The following assertions are equivalent:

(i) $\dot{X}=F(X)$ is complete;

(ii) $F$ is quadratic-affine or the differential equation $\dot{X}=F(X)$ is equivalent, by a linear change of coordinates, to a system

$$
\dot{x}=y(a x+b y), \quad \dot{y}=y(c x+d y),
$$

where $(a+d)^{2}-4(a d-b c)<0$.

\section{Complete left invariant pseudo Riemannian metrics defined on 3-dimensional unimodular Lie groups}

The Lie bracket of a 3-dimensional Lie algebra can be described using the vector product $\times$ corresponding to the positive definite scalar product $\langle\cdot, \cdot\rangle$ and to an orientation as follows [8]:

$$
[X, Y]=L(X \times Y),
$$

where $L \in \operatorname{End}(\mathcal{G})$.

If the group is unimodular, i.e. $\operatorname{tr}\left(\operatorname{ad}_{X}\right)=0$ for all $X \in \mathcal{G}$, then $L$ is $\langle\cdot, \cdot\rangle$-symmetric. Hence $\mathcal{G}$ has a $\langle\cdot, \cdot\rangle$-orthonormal basis of eigenvectors of $L, \mathcal{B}=\left(E_{1}, E_{2}, E_{3}\right)$, that is

$$
\left[E_{2}, E_{3}\right]=\alpha_{1} E_{1}, \quad\left[E_{3}, E_{1}\right]=\alpha_{2} E_{2}, \quad\left[E_{1}, E_{2}\right]=\alpha_{3} E_{3} .
$$

The signature of the eigenvalues characterizes the Lie algebra as is shown in the following diagram

\begin{tabular}{|c||c|c|c|}
\hline & signature of $\alpha_{1}, \alpha_{2}, \alpha_{3}$ & corresponding Lie group & type \\
\hline (a) & $0,0,0$ & $\mathbb{R} \oplus \mathbb{R} \oplus \mathbb{R}$ & Abelian \\
\hline (b) & $+, 0,0$ & Heisenberg & nilpotent \\
\hline (c) &,,+++ & $\mathrm{O}(3)$ & compact \\
\hline (d) &,,++ 0 & $\mathrm{E}(2)$ & solvable \\
\hline (e) &,,+- 0 & $\mathrm{E}(1,1)$ & solvable \\
\hline (f) &,,++- & $\mathrm{Sl}(2, \mathbb{R})$ & semisimple \\
\hline
\end{tabular}

Theorem 2. The following assertions are equivalent for left invariant pseudo-Riemannian metrics defined on 3-dimensional unimodular Lie groups

(i) the Euler equation is complete;

(ii) all geodesics that run on 1-parameter subgroups are complete;

(iii) the Euler field has no idempotents.

Notice that, by Remark $1,($ ii $) \Rightarrow($ iii $)$ since an idempotent of the Euler equation produces an incomplete geodesic that runs on a 1-parameter subgroup. Hence the only implication that requires proof is $(i i i) \Rightarrow(i)$.

In the first four cases, all pseudo-Riemannian metrics are complete, the first three being widely known:

(a) the Euler equation for a left invariant pseudo-Riemannian metric on an Abelian Lie group is complete; 
(b) the Euler equation for a left invariant pseudo-Riemannian metric on Heisenberg group is linearizable;

(c) the Euler equation for a left invariant pseudo-Riemannian metric on a compact Lie group has a definite quadratic first integral: the Killing form.

The proof of Theorem 2 consists on considerations of case by case situations.

Proposition 2. Every left invariant pseudo-Riemannian metric on $\mathrm{E}(2)$ is complete.

Proof. In a suitable basis, the Lie bracket is given by the relations:

$$
\left[E_{2}, E_{3}\right]=0, \quad\left[E_{3}, E_{1}\right]=E_{2}, \quad\left[E_{1}, E_{2}\right]=E_{3}
$$

and the Euler equation is

$$
\dot{\xi}_{1}=x_{3} \xi_{2}-x_{2} \xi_{3}, \quad \dot{\xi}_{2}=x_{1} \xi_{3}, \quad \dot{\xi}_{3}=-x_{1} \xi_{2}
$$

Since the semi-definite quadratic $\xi_{2}^{2}+\xi_{3}^{2}$ is a first integral of the Euler vector field, by Lemma 1 the Euler vector field has no idempotents.

We split the proof of the completeness of Euler vector field in two cases:

i) If the pseudo-Riemannian metric $\langle\cdot, \cdot\rangle$ is degenerate on the vector space spanned by $E_{2}, E_{3}$, then $x_{1}$ does not depend on $\xi_{1}$ and the two last equations of the Euler equation lie in the $\xi_{2} \xi_{3}$ plane. This planar system of equations has a positive definite quadratic first integral, hence it is complete. The first equation is of the form

$$
\dot{\xi}_{1}=f(t) \xi_{1}+g(t)
$$

and its solution is defined for all values of $t$ since $f$ and $g$ are so.

ii) If the pseudo-Riemannian metric $\langle\cdot, \cdot\rangle$ is non degenerate on the vector space spanned by $E_{2}, E_{3}$, then there is a basis of $\mathrm{E}(2)$ with $E_{1} \perp_{\langle\cdot, \cdot\rangle} \operatorname{Vect}\left\{E_{2}, E_{3}\right\}$ that satisfies the same relations for the bracket stated in equation (4). Then the first integral $\xi_{2}^{2}+\xi_{3}^{2}$ and the energy, that in this basis has the form $\xi_{1}^{2}+a \xi_{2}^{2}+b \xi_{2} \xi_{3}+c \xi_{3}^{2}$, can be combined to get a positive definite first integral. Hence the solutions of the Euler equation are complete.

In [6] another proof of Proposition 2 and an alternative statement and proof of Proposition 3 are provided.

Proposition 3. If the Euler product of a pseudo-Riemannian metric defined on E(1,1) has no idempotents then the Euler equation has a definite quadratic first integral, and hence such a metric is complete.

Proof. In a suitable basis, the Lie bracket is given by the relations

$$
\left[E_{1}, E_{2}\right]=E_{2}, \quad\left[E_{1}, E_{3}\right]=-E_{3}, \quad\left[E_{2}, E_{3}\right]=0
$$

and the Euler equation is

$$
\dot{\xi}_{1}=-x_{2} \xi_{2}+x_{3} \xi_{3}, \quad \dot{\xi}_{2}=x_{1} \xi_{2}, \quad \dot{\xi}_{3}=-x_{1} \xi_{3} .
$$

Besides the energy: $\left\langle\Phi^{-1} \xi, \Phi^{-1} \xi\right\rangle=x_{1} \xi_{1}+x_{2} \xi_{2}+x_{3} \xi_{3}$, the Euler vector field has $\xi_{2} \xi_{3}$ as a first integral. If the Euler vector field has no idempotents, the pseudo-Riemannian metric is non degenerate on $[\mathcal{G}, \mathcal{G}]=\operatorname{Vect}\left\{E_{2}, E_{3}\right\}$. In fact, for a pseudo-Riemannian metric which is degenerate on $[\mathcal{G}, \mathcal{G}], x_{1}$ does not depend on $\xi_{1}$ and any $\xi_{2}$ such that $x_{1}=1$ for $\xi_{3}=0$ gives an idempotent of the Euler vector field. 
Hence, if the Euler vector field has no idempotents, there exists $e_{1} \perp_{\langle\cdot, \cdot\rangle}[\mathcal{G}, \mathcal{G}]$ such that the bracket relations given by equation (5) are still satisfied, and the energy in these coordinates is given by:

$$
\lambda \xi_{1}^{2}+\mu \xi_{2}^{2}+2 c \xi_{2} \xi_{3}+\nu \xi_{3}^{2}
$$

Taking $\xi_{2}=0$, the equation for idempotents becomes

$$
\xi_{1}=x_{3} \xi_{3}=\nu \xi_{3}^{2}, \quad \xi_{3}=-x_{1} \xi_{3}=-\lambda \xi_{1} \xi_{3} .
$$

which has a solution if and only if $\lambda \mu<0$. Hence, if the Euler equation has no idempotents $\lambda \mu>0$. Analogously $\lambda \nu>0$. Since $\xi_{2} \xi_{3}$ is a first integral

$$
\lambda \xi_{1}^{2}+\mu \xi_{2}^{2}+\nu \xi_{3}^{2}
$$

is a first integral which is a definite quadratic form.

Proposition 4. If the Lax pair vector field for a pseudo-Riemannian metric on $\operatorname{Sl}(2, \mathbb{R})$ has no idempotents then it is complete.

In order to deal with $\mathrm{Sl}(2, \mathbb{R})$ some previous considerations are needed. The Killing form $\mathbf{k}$ of $\operatorname{sl}(2, \mathbb{R})$ is a quadratic form of index $(2,1)$, and induces a bi-invariant Lorentz metric on $\mathrm{Sl}(2, \mathbb{R})$. Thus the completeness of a left invariant pseudo-Riemannian metric is equivalent to the completeness of the Lax pair. We express this equation in two adapted basis (all non stated products are obtained either by symmetry or by antisymmetry):

(A) given a k-orthonormal basis, i.e. an ordered basis $\left(E_{1}, E_{2}, E_{3}\right)$, such that

$$
1=k\left(E_{1}, E_{1}\right)=k\left(E_{2}, E_{2}\right)=-k\left(E_{3}, E_{3}\right),
$$

the Lie bracket is given by the table

\begin{tabular}{c|c|c|c}
{$[]$,} & $E_{1}$ & $E_{2}$ & $E_{3}$ \\
\hline$E_{1}$ & 0 & $-\kappa E_{3}$ & $-\kappa E_{2}$ \\
\hline$E_{2}$ & & 0 & $\kappa E_{1}$ \\
\hline$E_{3}$ & & & 0
\end{tabular}

and matrix of $\operatorname{ad}_{X}$ is

$$
\operatorname{ad}_{X}=\kappa\left(\begin{array}{ccc}
0 & -x_{3} & x_{2} \\
x_{3} & 0 & -x_{1} \\
x_{2} & -x_{1} & 0
\end{array}\right)
$$

(B) given a k-hyperbolic basis, i.e. an ordered basis $\left(E_{1}, E_{2}, E_{3}\right)$, such that

$$
k\left(E_{1}, E_{1}\right)=k\left(E_{2}, E_{3}\right)=1,
$$

the Lie bracket is given by

\begin{tabular}{c|c|c|c}
{$[]$,} & $E_{1}$ & $E_{2}$ & $E_{3}$ \\
\hline$E_{1}$ & 0 & $\kappa E_{2}$ & $-\kappa E_{3}$ \\
\hline$E_{2}$ & & 0 & $\kappa E_{1}$ \\
\hline$E_{3}$ & & & 0
\end{tabular}


and $\operatorname{ad}_{X}$ has the matrix

$$
\operatorname{ad}_{X}=\kappa\left(\begin{array}{ccc}
0 & -x_{3} & x_{2} \\
-x_{2} & x_{1} & 0 \\
x_{3} & 0 & -x_{1}
\end{array}\right)
$$

where $\kappa=k\left(\left[E_{1}, E_{2}\right], E_{3}\right)$. Since it is possible to take care of a constant by a linear change of coordinates, it will be omitted in what follows.

As was said before, the proof will consist in dealing with each case of the Jordan form of $u$.

Lemma 2. If $u$ has exactly one eigendirection then the Lax pair vector field has always idempotents.

Proof. Let $E$ be an eigenvector. There are two possibilities: either $\mathbf{k}(E, E) \neq 0$ or $\mathbf{k}(E, E)=0$. Assume with no loss of generality that the eigenvalue is 1 .

Suppose first that $E$ is an eigenvector such that $\mathbf{k}(E, E) \neq 0$. Then $\mathbf{k}(E, E)>0$. In fact, under the hypothesis, the subspace $E^{\perp}$ is a 2-dimensional non degenerate subspace which is $u$ invariant. If $\mathbf{k}(E, E)<0, \mathbf{k}$ is positive definite on $E^{\perp}$. Thus $u$ is diagonalizable on $E^{\perp}$, which contradicts the assumption.

Let $E_{1}$ be an eingenvector such that $\mathbf{k}\left(E_{1}, E_{1}\right)=1$, and $E_{2}, E_{3} \in E^{\perp}$ such that $\mathbf{k}\left(E_{2}, E_{2}\right)=$ $-\mathbf{k}\left(E_{3}, E_{3}\right)=1, \mathbf{k}\left(E_{2}, E_{3}\right)=0$. The matrix of $u$ and of $u^{-1}$ are:

$$
\left(\begin{array}{ccc}
1 & 0 & 0 \\
0 & \alpha & \beta \\
0 & -\beta & \delta
\end{array}\right), \quad \frac{1}{\Delta}\left(\begin{array}{ccc}
\Delta & 0 & 0 \\
0 & \delta & -\beta \\
0 & \beta & \alpha
\end{array}\right),
$$

where $\Delta=\beta^{2}+\alpha \delta$. Notice that in this case the characteristic polynomial of $u, p_{u}(x)=(x-1)$ $(x-(\alpha+\delta) x+\Delta)$, has one simple real root. Hence $\Delta>0$ and $(\alpha-\delta)^{2}-4 \beta^{2}<0$. The Lax pair is given by:

$$
\begin{aligned}
& \dot{x}_{1}=\frac{1}{\Delta}\left(\beta x_{2}^{2}+(\alpha-\delta) x_{2} x_{3}+\beta x_{3}^{2}\right), \\
& \dot{x}_{2}=\frac{x_{1}}{\Delta}\left(-\beta x_{2}+(\Delta-\alpha) x_{3}\right), \\
& \dot{x}_{3}=\frac{x_{1}}{\Delta}\left((\Delta-\delta) x_{2}+\beta x_{3}\right) .
\end{aligned}
$$

If $\left(x_{1}, x_{2}, x_{3}\right)$ is an idempotent of the equation then $x_{1} \neq 0$ and $\Delta / x_{1}$ is an eigenvalue for

$$
\left(\begin{array}{cc}
-\beta & \Delta-\alpha \\
\Delta-\delta & \beta
\end{array}\right)
$$

Since the characteristic polynomial of this matrix is $x^{2}-\left(\beta^{2}+(\Delta-\alpha)(\Delta-\delta)\right)$, then the planar system has idempotents if and only if

$$
0<\beta^{2}+(\Delta-\alpha)(\Delta-\delta)=\Delta\left(\beta^{2}+\alpha \delta+1-(\alpha+\delta)\right)
$$

or if and only if $\beta^{2}+\alpha \delta+1-(\alpha+\delta)>0$, because $\Delta>0$. This inequality holds since

$$
\beta^{2}+\alpha \delta>\frac{1}{4}(\alpha+\delta)^{2} \geq(\alpha+\delta)-1 .
$$

Hence the planar system has two eigendirections corresponding to two eigenvalues of different sign. Finally to produce an idempotent for the Lax pair, it is necessary to solve the equation

$$
\frac{\Delta}{x_{1}}=\frac{\Delta^{2}}{\beta x_{2}^{2}+(\alpha-\delta) x_{2} x_{3}+\beta x_{3}^{2}},
$$


where $\Delta / x_{1}$ is an eigenvalue and $\left(x_{2}, x_{3}\right)$ a corresponding eigenvector. Since the quadratic form $\beta x_{2}^{2}+(\alpha-\delta) x_{2} x_{3}+\beta x_{3}^{2}$ is definite, this equation has always a solution.

Now suppose that $\mathbf{k}(E, E)=0$. Let $E_{3}$ be an eigenvector. By hypothesis, $\mathbf{k}\left(E_{3}, E_{3}\right)=0$. Let $E_{2}$ be such that $\mathbf{k}\left(E_{3}, E_{2}\right)=1$ and $\mathbf{k}\left(E_{2}, E_{2}\right)=0$. Choose $E_{1} \mathbf{k}$-orthogonal to $E_{2}$ and $E_{3}$, with $\mathbf{k}\left(E_{1}, E_{1}\right)=1$, which is possible because of the signature of $\mathbf{k}$. Hence we obtain a $\mathbf{k}$ hyperbolic basis $\left(E_{1}, E_{2}, E_{3}\right)$. Since $u$ is $\mathbf{k}$-symmetric, the matrix of $u$ in the basis constructed above is

$$
\left(\begin{array}{lll}
\gamma & a & 0 \\
0 & 1 & 0 \\
a & b & 1
\end{array}\right),
$$

whose characteristic polynomial is $p_{u}(x)=(x-\gamma)(x-1)^{2}$. Since $u$ has only one eigendirection, $\gamma=1$ and the matrix of $u^{-1}$ is

$$
\left(\begin{array}{ccc}
1 & -a & 0 \\
0 & 1 & 0 \\
-a & a^{2}-b & 1
\end{array}\right)
$$

The Lax pair is given by:

$$
\begin{aligned}
& \dot{x}_{1}=-a x_{1} x_{2}+\left(a^{2}-b\right) x_{2}^{2}+(b-1) x_{2} x_{3}, \\
& \dot{x}_{2}=a x_{2}^{2}, \\
& \dot{x}_{3}=a x_{1}^{2}-\left(a^{2}-b\right) x_{1} x_{2}-a x_{2} x_{3} .
\end{aligned}
$$

Notice that the hypothesis imply that $a \neq 0$. An idempotent is produced by setting $x_{2}=1 / a$ and solving the other two equations, with this value for $x_{2}$.

Lemma 3. Let $u$ be diagonalizable. Then the pseudo-Riemannian metric on $\mathrm{Sl}(2, \mathbb{R})$ defined by $u$ has a definite quadratic first integral (and hence is complete) provided that the Lax pair vector field has no idempotents.

Proof. If $u$ is diagonalizable then there is a $k$-orthonormal basis $E_{1}, E_{2}, E_{3}$ with $E_{1}, E_{2}, E_{3}$ eigenvectors of $u$. In fact, if $u$ has one eigenvalue with eigenspace of dimension 3 , then $u$ is a multiple of the identity and the assertion follows trivially. If $u$ has three different eigenvalues, then the proof is straightforward. Assume $u$ has two eigenvalues and that one of them has eigenspace of dimension 2 , i.e. there exists a basis $\left\{E_{1}, E_{2}, E_{3}\right\}$ such that $u\left(E_{1}\right)=\lambda E_{1}, u\left(E_{2}\right)=$ $\lambda E_{2}, u\left(E_{3}\right)=\mu E_{3}, \lambda \neq \mu$. Then the restriction of $u$ to the eigenspace corresponding to $\lambda$ is a multiple of the identity. Since $\mathbf{k}\left(E_{1}, E_{3}\right)=\mathbf{k}\left(E_{2}, E_{3}\right)=0$, then $\mathbf{k}\left(E_{3}, E_{3}\right) \neq 0$. Hence the $\mathbf{k}$-non degenerate subspace $\operatorname{Vect}\left\{E_{3}\right\}$ is invariant by $u$. This implies that the eigenspace $\operatorname{Vect}\left\{E_{1}, E_{2}\right\}=\operatorname{Vect}\left\{E_{3}\right\}^{\perp}$ is $\mathbf{k}$-non degenerate. And the assertion follows.

Hence the matrix of $u^{-1}$ in the $\mathbf{k}$-orthonormal basis of eigenvectors is given by

$$
\left(\begin{array}{ccc}
\lambda_{1} & 0 & 0 \\
0 & \lambda_{2} & 0 \\
0 & 0 & \lambda_{3}
\end{array}\right),
$$

where $\lambda_{1}^{-1}, \lambda_{2}^{-1}$ and $\lambda_{3}^{-1}$ are the eigenvalues of $u$. The Lax pair is given by

$$
\begin{aligned}
& \dot{x}_{1}=\left(\lambda_{3}-\lambda_{2}\right) x_{2} x_{3}=a x_{2} x_{3}, \\
& \dot{x}_{2}=\left(\lambda_{1}-\lambda_{3}\right) x_{1} x_{3}=b x_{1} x_{3}, \\
& \dot{x}_{3}=\left(\lambda_{1}-\lambda_{2}\right) x_{1} x_{2}=(a+b) x_{1} x_{2},
\end{aligned}
$$


where $a=\lambda_{3}-\lambda_{2}, b=\lambda_{1}-\lambda_{3}$. The equation for idempotents is:

$$
x_{1}=a x_{2} x_{3}, \quad x_{2}=b x_{1} x_{3}, \quad x_{3}=(a+b) x_{1} x_{2},
$$

which is equivalent to:

$$
b(a+b) x_{1}^{2}=a(a+b) x_{2}^{2}=a b x_{3}^{2} .
$$

Equation (6) has a non trivial solution if and only if $a, b$ and $a+b$ have the same signature, that is, if and only if $a b>0$. Hence the Euler product has no non trivial idempotents if and only if $a b<0$ or $a b(a+b)=0$. If $a b(a+b)=0$ the Lax pair is linearizable. Assume $a b<0$. Clearly

$$
b x_{1}^{2}-a x_{2}^{2}
$$

is a first integral. This quadratic, which is a semi-definite quadratic form, and the quadratic first integral $x_{1}^{2}+x_{2}^{2}-x_{3}^{2}$ can be combined into a quadratic positive definite first integral.

Lemma 4. If u has two linearly independent eigenvectors corresponding to two different eigenvalues then the left invariant pseudo-Riemannian metric on $\mathrm{Sl}(2, \mathbb{R})$ defined by $u$ is complete whenever the Lax pair vector field has no idempotents.

Proof. Let $p_{u}(x)=(x-\alpha)^{2}(x-\beta)$ be the characteristic polynomial of $u, E$ an eigenvector of $u$ corresponding to the simple eigenvalue $\beta$. Denote by $V$ the subspace $\left\{x \in \mathcal{G}:(u-\alpha \text { Id })^{2} x=0\right\}$. Then $V$ and the subspace generated by $E$ are $u$ invariant and $E \perp_{k} V$. The last assertion follows from the following calculation: when $x \in V$,

$$
u^{2}(x)-2 \alpha u(x)+\alpha^{2} x=0
$$

and

$$
\begin{aligned}
\alpha^{2} \mathbf{k}(E, x) & =-\mathbf{k}\left(E, u^{2}(x)-2 \alpha u(x)\right)=-\mathbf{k}\left(E, u^{2}(x)\right)+2 \alpha \mathbf{k}(E, u(x)) \\
& =-\beta^{2} k(E, x)+2 \alpha \beta k(E, x)
\end{aligned}
$$

since $u$ is $\mathbf{k}$-symmetric. Hence

$$
\left(\alpha^{2}+\beta^{2}-2 \alpha \beta\right) \mathbf{k}(E, x)=0
$$

and $\mathbf{k}(E, x)=0$. (By hypothesis, $\alpha \neq \beta$.)

For every $E^{\prime} \in V$ eigenvector corresponding to $\alpha, \mathbf{k}\left(E^{\prime}, E^{\prime}\right)=0$ because if $\mathbf{k}\left(E^{\prime}, E^{\prime}\right) \neq 0$, then $\operatorname{Vect}\left\{E, E^{\prime}\right\}$ is a $u$ invariant, $\mathbf{k}$ - non degenerate subspace. Hence $\operatorname{Vect}\left\{E, E^{\prime}\right\}^{\perp_{\mathbf{k}}}$ is $u$ invariant of dimension 1. This implies the existence of an eigenvector $E^{\prime \prime} \notin \operatorname{Vect}\left\{E, E^{\prime}\right\}$ which by hypothesis is not possible.

The subspace $V$ is $\mathbf{k}$-non degenerate, of dimension 2 and has an isotropic vector. Hence it is a hyperbolic plane. Since $\mathbf{k}$ is of index $(2,1), \mathbf{k}(E, E)>0$. Let $E_{1}=E / \sqrt{\mathbf{k}(E, E)}$.

Let $E_{2}, E_{3} \in V$ such that

$$
\begin{aligned}
& \mathbf{k}\left(E_{2}, E_{2}\right)=0=\mathbf{k}\left(E_{3}, E_{3}\right), \quad \mathbf{k}\left(E_{2}, E_{3}\right)=1 \quad \text { and } \\
& u\left(E_{2}\right)=\alpha E_{2}, \quad u\left(E_{3}\right)=\gamma E_{2}+\alpha E_{3},
\end{aligned}
$$

where $0 \neq \gamma=\left\langle E_{3}, E_{3}\right\rangle$. In this $\mathbf{k}$-hyperbolic basis, the matrix of $u^{-1}$ is

$$
\left(\begin{array}{ccc}
b & 0 & 0 \\
0 & a & -\gamma a^{2} \\
0 & 0 & a
\end{array}\right),
$$

where $a=\alpha^{-1}$ and $b=\beta^{-1}$. 
The Lax pair associated to the pseudo-Riemannian metric is

$$
\begin{aligned}
& \dot{x}_{1}=\gamma a^{2} x_{3}^{2}, \\
& \dot{x}_{2}=-\gamma a^{2} x_{1} x_{3}+(a-b) x_{1} x_{2}, \\
& \dot{x}_{3}=(b-a) x_{1} x_{3} .
\end{aligned}
$$

The first and third equations conform a planar system which has idempotents if and only if

$$
\gamma(b-a)>0 .
$$

The idempotent obtained produces an idempotent for the Lax pair vector field. Then, if the Lax pair vector field has no idempotent we get a semi-definite quadratic first integral for the whole system and a definite quadratic first integral for the planar system:

$$
(a-b) x_{1}^{2}+\gamma a^{2} x_{3}^{2} .
$$

Thus the planar system is complete. The second equation is of the type

$$
\dot{x}_{2}=f(t)+g(t) x_{2} .
$$

where $f, g$ are defined for all $t$, therefore it is also complete.

Lemma 5. If u has two linearly independent eigenvectors corresponding to the same eigenvalue, then the Lax pair associated to $u$ on $\mathrm{Sl}(2, \mathbb{R})$ is linearizable.

Proof. Assuming that $u$ has two eigendirections and exactly one eigenvalue, there is a basis of $\mathcal{G}, E_{1}, E_{2}, E_{3}$, such that

$$
M_{\mathcal{B}}(u)=\left(\begin{array}{ccc}
\alpha & 1 & 0 \\
0 & \alpha & 0 \\
0 & 0 & \alpha
\end{array}\right) .
$$

We have that $E_{1}^{\perp}=\operatorname{Vect}\left\{E_{1}, E_{3}\right\}$. In fact, since $u$ is $\mathbf{k}$-symmetric,

$$
\begin{array}{ccc}
\mathbf{k}\left(E_{1}, u\left(E_{2}\right)\right) & = & \mathbf{k}\left(u\left(E_{1}\right), E_{2}\right) \\
\text { "I } & & \text { ॥ } \\
\mathbf{k}\left(E_{1}, E_{1}\right)+a \mathbf{k}\left(E_{1}, E_{2}\right) & = & a \mathbf{k}\left(E_{1}, E_{2}\right)
\end{array}
$$

which implies that $\mathbf{k}\left(E_{1}, E_{1}\right)=0$, and

$$
\begin{array}{ccc}
\mathbf{k}\left(E_{3}, u\left(E_{2}\right)\right) & = & \mathbf{k}\left(u\left(E_{3}\right), E_{2}\right) \\
\mathbf{k}\left(E_{3}, E_{1}\right)+a \mathbf{k}\left(E_{3}, E_{2}\right) & = & a \mathbf{k}\left(E_{3}, E_{2}\right)
\end{array}
$$

which implies that $\mathbf{k}\left(E_{1}, E_{3}\right)=0$.

Hence

$$
\mathbf{k}\left(E_{1}, E_{2}\right) \neq 0 \text {. }
$$

The subspace $V=\operatorname{Vect}\left\{E_{1}, E_{2}\right\}$ is non degenerate and $u$ invariant. Thus $V^{\perp_{\mathbf{k}}}$ is a onedimensional subspace, non degenerate and $u$ invariant.

The Lax pair associated to the pseudo-Riemannian metric is

$$
\dot{x}_{1}=a^{2} x_{3}^{2}, \quad \dot{x}_{2}=-a^{2} x_{1} x_{3}, \quad \dot{x}_{3}=0,
$$

which is linearizable. 
We can summarize the situation in $\operatorname{Sl}(2, \mathbb{R})$ with the following proposition

Proposition 5. A left invariant pseudo-Riemannian metric in $\mathrm{Sl}(2, \mathbb{R})$ with associated isomorphism $u$ and minimal polynomial $q_{u}(x)$, is complete if and only if

(i) $q_{u}(x)$ has degree less than or equal to 2. In this case the Euler equation is linearizable;

(ii) $q_{u}(x)=\left(x-\alpha_{1}\right)\left(x-\alpha_{2}\right)\left(x-\alpha_{3}\right)$ where $\alpha_{i} \neq \alpha_{j}$ if $i \neq j$ and

$$
\left(\frac{1}{\alpha_{3}}-\frac{1}{\alpha_{2}}\right)\left(\frac{1}{\alpha_{3}}-\frac{1}{\alpha_{1}}\right)>0
$$

where $\alpha_{3}$ is the eigenvalue of $u$ corresponding to an eigenvector $E$ such that $\mathbf{k}(E, E)<0$. In this case, the Euler equation of a complete left invariant pseudo-Riemannian metric has a definite quadratic first integral;

(iii) $q_{u}(x)=\left(x-\alpha_{1}\right)\left(x-\alpha_{2}\right)^{2}$ and

$$
\left\langle E_{3}, E_{3}\right\rangle\left(\frac{1}{\alpha_{2}}-\frac{1}{\alpha_{1}}\right)>0
$$

where $E_{3}$ is a cyclic vector associated to $\alpha_{2}$.

\section{Some examples and remarks}

It is worth noting that, except for the case $\mathrm{E}(2)$ and the case in Proposition 5(iii) we have shown that a left invariant pseudo-Riemannian metric on a non unimodular Lie group is complete if and only if either the Euler equation is linearizable or has a definite quadratic first integral. The following example shows that this is not the case for $\mathrm{E}(2)$ (and an example can be found in the same way in $\mathrm{Sl}(2, \mathbb{R})$ ). However in these cases the differential equation is of the type:

$$
\dot{x}_{1}=X_{1}\left(x_{1}, x_{2}\right), \quad \dot{x}_{2}=X_{2}\left(x_{1}, x_{2}\right), \quad \dot{x}_{3}=X_{3}\left(x_{1}, x_{2}, x_{3}\right)
$$

$\left(X_{1}, X_{2}, X_{3}\right.$ quadratic polynomials) where the two first equations have a definite quadratic first integral.

Example 1. Consider on E(2) the pseudo-Riemannian metric given by

$$
-x_{1}^{2}+x_{2}^{2}+2 x_{2} x_{3} .
$$

in the basis considered in Proposition 2. The corresponding Euler equation is

$$
\dot{\xi}_{1}=\xi_{1} \xi_{2}, \quad \dot{\xi}_{2}=\xi_{3}^{2}, \quad \dot{\xi}_{3}=-\xi_{2} \xi_{3}
$$

This equation is complete and is not linearizable. However $\xi_{1}$ is not bounded, hence no first integral can have bounded level surfaces.

In [4] a characterization of the complete pseudo-Riemannian metrics on $\mathrm{Sl}(2, \mathbb{R})$ is given:

Theorem $^{1}$. The Lorentzian left invariant pseudo-Riemannian metric on $\mathrm{Sl}(2, \mathbb{R})$ is defined by the isomorphism $\phi$ is complete only in the two following cases:

\footnotetext{
${ }^{1}$ Theorem. Si la métrique invariante à gauche sur $\mathrm{Sl}(2, \mathbb{R})$ définie par l'endomorphisme $\phi$ est lorentzienne, elle n'est complète que dans les deux cas suivants:

1) $\phi$ admet un sous-espace propre de dimension 2 au moins;

2) $\phi$ est diagonalizable sur $\mathbb{R}$, admet deux valeurs propres opposeées, et les vecteurs propres associés engendrent un plan lorentzien.
} 
1) $\phi$ has an eigenspace of dimension at least two;

2) $\phi$ is diagonalizable over the real numbers, has two eigenvalues of different sign and the eigenvectors associated to these eigenvalues generate a Lorentzian plane.

However, the following examples show that this characterization is not correct.

Example 2. Consider on $\operatorname{Sl}(2, \mathbb{R})$ the left invariant Lorentzian metric given in a k-orthonormal basis $\mathcal{B}=\left(E_{1}, E_{2}, E_{3}\right)$ by

$$
x_{1}^{2}+x_{2}^{2}+x_{2} x_{3} .
$$

In the $\mathbf{k}$-hyperbolic basis deduced from $\mathcal{B}, \mathcal{B}^{\prime}=\left(E_{1},(1 / \sqrt{2})\left(E_{2}+E_{3}\right),(1 / \sqrt{2})\left(E_{2}-E_{3}\right)\right)$, the $\mathbf{k}$-symmetric isomorphism $u$ that corresponds to the Lorentzian metric is given by the matrix

$$
M_{\mathcal{B}^{\prime}}(u)=\left(\begin{array}{ccc}
1 & 0 & 0 \\
0 & 1 / 2 & 1 \\
0 & 0 & 1 / 2
\end{array}\right) .
$$

The characteristic polynomial of $u$ is $p_{u}(x)=(x-1 / 2)^{2}(x-1)$, with eigendirections $E_{1}$ and $E_{2}+E_{3}$. The Lax pair equation corresponding to this Lorentzian metric in $\mathcal{B}^{\prime}$ coordinates is

$$
\dot{x_{1}}=4 x_{3}^{2}, \quad \dot{x_{2}}=x_{1}\left(x_{2}-4 x_{3}\right), \quad \dot{x_{3}}=-x_{1} x_{3} .
$$

The planar system given by the first and third equations is complete. Hence the system is complete. Notice that $u$ has neither an eigenspace of dimension 2 nor is it diagonalizable.

Example 3. Let $u$ be the isomorphism with matrix

$$
M_{\mathcal{B}}(u)=\left(\begin{array}{ccc}
1 / 2 & 0 & 0 \\
0 & 1 / 3 & 0 \\
0 & 0 & 1
\end{array}\right) .
$$

The pseudo-Riemannian metric is given by:

$$
\langle x, x\rangle=\mathbf{k}(u(x), x)=\frac{1}{2} x_{1}^{2}+\frac{1}{3} x_{2}^{2}-x_{3}^{2}
$$

which is Lorentzian. Notice that all eigenvalues of $u$ are positive

The Lax pair corresponding to this pseudo-Riemannian metric is

$$
\dot{x}_{1}=-2 x_{2} x_{3}, \quad \dot{x}_{2}=x_{1} x_{3}, \quad \dot{x}_{3}=-x_{1} x_{2} .
$$

This equation has the following first integrals

$$
x_{1}^{2}+x_{2}^{2}-x_{3}^{2} \quad \text { and } \quad x_{2}^{2}+x_{3}^{2} .
$$

Hence the solutions are bounded and the equation is complete.

Example 4. Consider the pseudo-Riemannian metric in the same basis as before given by

$$
x_{1}^{2}-x_{2}^{2}+2 x_{3}^{2} .
$$

The isomorphism $u$ associated to this pseudo-Riemannian metric has $E_{1}, E_{2}$, and $E_{3}$ as eigenvectors with (respectively) eigenvalues $1,-1$ and 2 . Hence we have two opposite eigenvalues whose corresponding eigenvectors generate a Lorentzian plane. The Lax pair equation in this case is:

$$
\dot{x}_{1}=\frac{1}{2} x_{2} x_{3}, \quad \dot{x}_{2}=\frac{3}{2} x_{1} x_{3}, \quad \dot{x}_{3}=2 x_{1} x_{2} .
$$

Since $\left(\sqrt{\frac{1}{3}}, 1,2 \sqrt{\frac{1}{3}}\right)$ is an idempotent for the Lax pair vector field, the pseudo-Riemannian metric is not complete.

We have shown that for unimodular Lie groups of dimension 3 the simplest case for incompleteness occur. This is not the case in general as the following example shows. 
Example 5. Let $\mathcal{G}$ be a non unimodular Lie algebra de Lie of dimension 3 . Then there is a basis $\left(e_{1}, e_{2}, e_{3}\right)$, of $\mathcal{G}$ such that the Lie bracket is given by

\begin{tabular}{c|c|c|c}
{$[]$,} & $e_{1}$ & $e_{2}$ & $e_{3}$ \\
\hline$e_{1}$ & 0 & $\alpha e_{2}+\beta e_{3}$ & $\gamma e_{2}+\delta e_{3}$ \\
\hline$e_{2}$ & & 0 & 0 \\
\hline$e_{3}$ & & & 0
\end{tabular}

where $\alpha+\delta=2[8]$. For $\alpha=1 / 2, \delta=3 / 2$, and energy $2 \xi_{1}^{2}-\xi_{2} \xi_{3}$, the Euler equation is:

$$
\dot{\xi}_{1}=\xi_{2} \xi_{3}, \quad \dot{\xi}_{2}=\xi_{1} \xi_{2}, \quad \dot{\xi}_{3}=3 \xi_{1} \xi_{3} .
$$

Clearly the Euler vector field has no idempotents and the curve

$$
\xi(t)=\left(\frac{1}{\sqrt{2}} \frac{1}{1-\sqrt{2} t}, \frac{1}{(1-\sqrt{2} t)^{1 / 2}}, \frac{1}{(1-\sqrt{2} t)^{3 / 2}}\right)
$$

is an incomplete solution with initial condition $\xi(0)=(1 / \sqrt{2}, 1,1)$.

\section{Acknowledgements}

The authors wish to thank the anonymous referee for the careful reading and the pertinent observations that led, in particular, to a revision of the proof of Lemma 4 and to reformulate accordingly Proposition 5.

\section{References}

[1] Bromberg S., Medina A., Complétude de l'équation d'Euler, in Proceedings of the Colloquium in Tashkent "Algebra and Operator Theory" (September 29 - October 5, 1997, Tashkent), Editors Y. Khakimdjanov, M. Goze and S.A. Ayupov, Kluwer Acad. Publ., Dordrecht, 1998, 127-144.

[2] Bromberg S., Medina A., Completeness of homogeneous quadratic vector fields, Qual. Theory Dyn. Syst. 6 (2005), 181-185.

[3] Dumitrescu S., Zeghib A., Géométries Lorentziennes de dimension 3: classification et complétude, math.DG/0703846.

[4] Guediri M., Lafontaine J., Sur la complétude des varietés pseudo-Rimanniennes, J. Geom. Phys. 15 (1995), $150-158$.

[5] Guediri M., Sur la complétude des pseudo-métriques invariantes a gauche sur les groupes de Lie nilpotents, Rend. Sem. Math. Univ. Politec. Torino 52 (1994), 371-376.

[6] Guediri M., On completeness of left-invariant Lorentz metrics on solvable Lie groups, Rev. Mat. Univ. Complut. Madrid 9 (1996), 337-350.

[7] Kaplan J.L., Yorke J.A., Non associative real algebras and quadratic differential equations, Nonlinear Anal. 3 (1979), 49-51.

[8] Milnor J., Curvatures of left invariant metrics on Lie groups, Advances in Math. 21 (1976), 293-329. 